\title{
The Added Value of B-Flow Technique in Assessment of Lower Limb Arterial Lesions with Densely Calcified Atheromatous Plaques
}

\author{
Mahmoud H. ElShenawy ${ }^{\text {a }}$, Islam M. ElShazly ${ }^{\mathrm{b}}$, Hesham E. ElSheik ${ }^{\mathrm{b}}$
}

a

Department of Radiology, ElAgouza hospital, Cairo, Egypt.

b Department of Radiology, Benha faculty of medicine, Benha University, Egypt.

Correspondence to:Mahmoud H. ElShenawy, Department of Radiology, ElAgouza hospital, Cairo, Egypt.

Email:

mahmoud.shenawi@gmail.com

Received: 10 August 2020

Accepted: 5 April 2021

\begin{abstract}
:
Background: Diagnostic CTA is the gold standard for assessment of PAD. However, ultrasound is more desirable for being non-invasive, real time and using non-ionizing radiation. As such, GE healthcare has introduced "B-Flow" as a new ultrasound imaging mode that can overcome some limitations of CDU, especially densely calcified plaques. Aim: To assess the added value of B-flow technique in assessment of lower limb arterial lesions in presence of densely calcified atheromatous plaques. Patients and methods: Our study included 30 cases having densely calcified arterial atheromatous plaques. All subjects were examined by CDU with spectral analysis and BFI; and the results were compared to the gold standard CTA. Results: BFI has higher discrimination than CDU in presence of densely calcified plaques at the femoral segments. It is also better in detecting sites of collaterals take-off at the infra-popliteal segments. However, extensively calcified plaques, depth and edema represent
\end{abstract} major limitations. Conclusion: B-flow has the potential to improve US imaging in patients with PAD. However, it is not free of limitations. As such, we do not encourage the use of B-Flow as a sole ultrasound imaging mode, but as a complementary technique to use in situations where CDU findings are inconclusive or unclear.

Key words: PAD, B-Flow, BFI, dense calcifications, CDU.

List of abbreviations: CDU: color Doppler ultrasound, PAD: peripheral arterial disease, BFI: BFlow imaging, CTA: Computed tomography angiography, CFA: common femoral artery, SFA: superficial femoral artery, POPA: popliteal artery, PTA: posterior tibial artery, ATA: anterior tibial artery, DPA: dorsalis pedis artery, FOV: field of view, PPV: positive predictive value, NPV: negative predictive value. 


\section{Introduction}

Peripheral arterial disease (PAD) is a nearly pandemic condition that has the potential to cause loss of limb or even loss of life ${ }^{1}$. Diagnosing of PAD is based on careful history, physical examination and a variety of diagnostic tools. Arteriography has been considered the gold standard for the investigation of PAD as it provides anatomical details that cannot be obtained from other imaging techniques. However, arteriography is invasive, expensive, with limited access, and depends on usage of ionizing radiation or contrast agents. As such, it cannot be used as a screening modality and it may be associated with some morbidity. In addition to that, it has technical some limitations, such as inaccurate estimation of the hemodynamic effect of arterial lesions and detection of patency of distal arteries subjected to low flow ${ }^{1}$.

On the other hand, Color Doppler Ultrasound (CDU) is a good method for screening and follow-up, as well as for the definitive diagnosis of peripheral arterial disease $^{2}$. It is often more desirable for being non-invasive, non-ionizing radiation, real-time and economical. Besides that, with the usage of Pulsed-wave, CDU can show the exact flow velocity of each arterial segment and determine the degree of severity of the stenosis based on an analysis of the pulsed-wave Doppler spectral waveform $^{3}$. So, CDU can provide information not only about vessel wall, but also hemodynamic information. However, color images of blood flow can be seen to overwrite the vessel boundaries and calcified plaques might cause acoustic shadowing obscuring details ${ }^{2}$. Other limitations include angle dependence, aliasing, and difficulty in separating background noise from true flow in slowflow states in multilevel stenosis ${ }^{1}$. Hence comes the new technology "B-flow" introduced from GE Healthcare that has shown great potential for addressing these challenges $^{2}$.

B-Flow is a new, unexplored, non-Doppler US imaging mode that was developed trying to overcome the various limitations of CDU. It applies digitally encoded ultrasound (DEU) technology to directly visualize the flowing blood cells ${ }^{4}$. Its flow images are provided by highlighting the weak flow reflectors (red blood cells) and suppressing the signals from the surrounding stationary tissue ${ }^{4}$.

B-flow is exempt from movement, blooming 
and aliasing artifacts. It shows higher frame rate for hemodynamic imaging, and higher spatial resolution for vascular anatomy ${ }^{5}$. It can also simultaneously visualize the flowing blood and the surrounding stationary structures.

\section{Aim of Work}

To assess the added value of BFI in assessment of lower limb densely calcified arterial plaques, highlighting the potential advantages and limitations of this technique.

\section{Patients and Methods}

This is a prospective case-control study. It included 30 cases with densely calcified atheromatous plaques. The study was prospectively done at El Tahra radiology center, during the period from July 2018 to June 2020. Approvement was gotten from the ethics committee of the institute and informed consents were obtained from the patients.

\section{Inclusion Criteria}

- Patients with suboptimal US assessments caused by the densely calcified atheromatous plaques that obscure an arterial segment.

-All the patients underwent further assessment using CT angiography as gold standard.
-All the included patients approved to be included in this study.

\section{Exclusion Criteria}

-Patients lacking the required investigations (No CT scan was done).

-Patients who didn't follow up.

-Patients who didn't agree to be included in this study.

\section{Methodology}

All examinations were done by the same examiner. No patient preparation was requested.

A linear transducer with a variable ultrasound frequency of $7-12 \mathrm{MHz}$ is generally used, but a convex transducer with a lower frequency was used as well with thick patients and for evaluation of the iliac arteries in the pelvic cavity.

The examination is usually performed with the patient placed in the supine position. The patient's hip is generally abducted and externally rotated, and the knee is flexed like frog legs in order to easily approach the POPA in the popliteal fossa and the PTA in the medial calf.

We start the examination by visualizing the CFA at the inguinal region in a transverse plane then the transducer is rotated $90^{\circ}$ for longitudinal scanning. With B-mode, we 
assess the arterial wall for intima thickness, any lumen irregularities and the atheromatous plaques, especially the densely calcified ones. Then CDU is applied with color box tilt parallel to the arterial axis and the color gain is set as high as possible without displaying background noise, the vessel is then assessed for its homogenous color filling and flow direction, visualizing the color flow at the stenotic segments, looking for aliasing or any possible collaterals. After that we apply spectral Doppler with Doppler angle between $50^{\circ}$ and $60^{\circ}$ to assess blood flow regarding direction, velocity, and acceleration. Lastly, we use BFI to display blood flow signal at the stenotic segments, especially at the densely calcified plaques that cause acoustic shadowing artifacts, visualizing the residual patent lumen that couldn't be viewed by CDU, allowing Doppler velocities to be sampled in regions that were otherwise obscured by the acoustic shadowing artifact. Possible collaterals are scanned by B-Flow mode as well, because they are much easier to be detected by this mode, thanks to its extended FOV and angle independency.

The examination is continued by moving a transducer distally along the SFA on the medial aspect of the leg, down till the POPA at the popliteal fossa. The arteries were assessed by the same means as mentioned before using B-mode, CDU, Spectral Doppler and BFI.

As for the InfraPopliteal arteries, the PTA is examined from the ankle behind the medial malleolus and continued proximally along the medial aspect of the leg, and the ATA is examined from the ankle anterior to the talus neck and continued proximally, the transducer is traced from the anterior ankle to the dorsal foot as well to evaluate the DPA. All the InfraPopliteal arteries were examined by the same means as mentioned before using B-mode, CDU, Spectral Doppler and BFI.

Test results of $\mathrm{CDU}$ and $\mathrm{BFI}$ were first analyzed separately and each compared with the reference CTA. Results were interpreted by calculating sensitivity, specificity, positive predictive value (PPV) and negative predictive value (NPV).

\section{Equipment:}

-Ultrasound was performed using LOGIQ S7 ultrasound system which supports B-flow and can combine real time B-mode imaging system with pulsed and continuous wave Doppler facilities together.

-CT was performed by using 16 channels MSCT helical, Toshiba. 


\section{Results}

The study included 30 patients, 22 males and 8 females, all of them had densely calcified atheromatous plaques scattered along the arterial system with different degrees of stenosis. 330 different arterial segments were

Table 1: The CFA results examined by CDU with spectral analysis and $\mathrm{BFI}$, and the results were compared to the gold standard CTA.

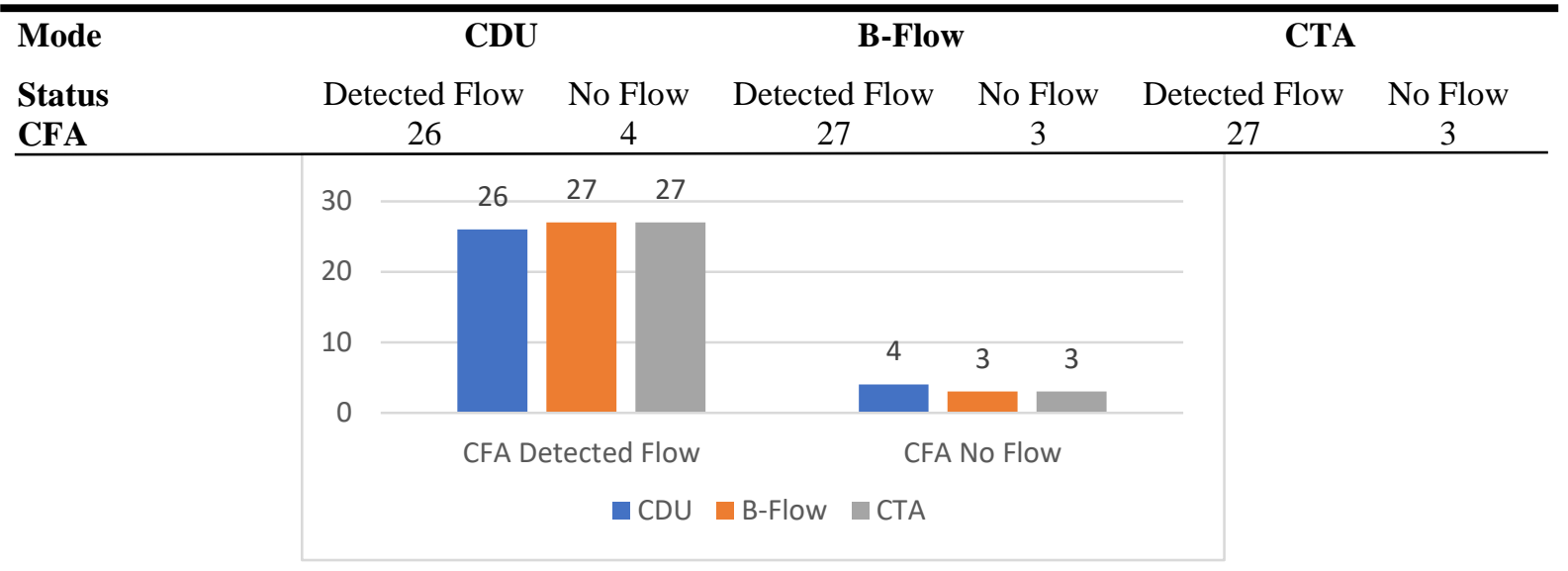

Graph 1. The CFA results

- The incidence of detected blood flow at the CFA was $86.6 \%$ by CDU and $90 \%$ by both BFI and CTA.

- The incidence of no flow at the CFA occlusion was $13.3 \%$ by CDU and $10 \%$ by both BFI and CTA.

- The sensitivity of CDU at the CFA in our study was $100 \%$, the specificity was
$96.3 \%$, the positive predictive value was $75 \%$ and the negative predictive value was $100 \%$.

- The sensitivity of BFI at the CFA in our study was $100 \%$, the specificity was $100 \%$, the positive predictive value was $100 \%$ and the negative predictive value was $100 \%$. 
Benha medical journal, vol. 38, issue 1, 2021

Table 2. The results of the different segments of the SFA

\begin{tabular}{lcccccc}
\hline $\begin{array}{l}\text { Mode } \\
\text { Status }\end{array}$ & \multicolumn{2}{c}{ CDU } & \multicolumn{2}{c}{ B-Flow } & \multicolumn{2}{c}{ CTA } \\
& Detected Flow & No Flow & Detected Flow & No Flow & Detected Flow & No Flow \\
$\begin{array}{l}\text { SFA } \\
\text { Proximal 1/3 }\end{array}$ & 27 & 3 & 25 & 5 & 27 & 3 \\
$\begin{array}{l}\text { SFA } \\
\text { Middle 1/3 }\end{array}$ & 21 & 9 & 23 & 7 & 27 & 3 \\
$\begin{array}{l}\text { SFA } \\
\text { Distal 1/3 }\end{array}$ & 27 & 3 & 27 & 3 & 27 & 3 \\
\hline
\end{tabular}

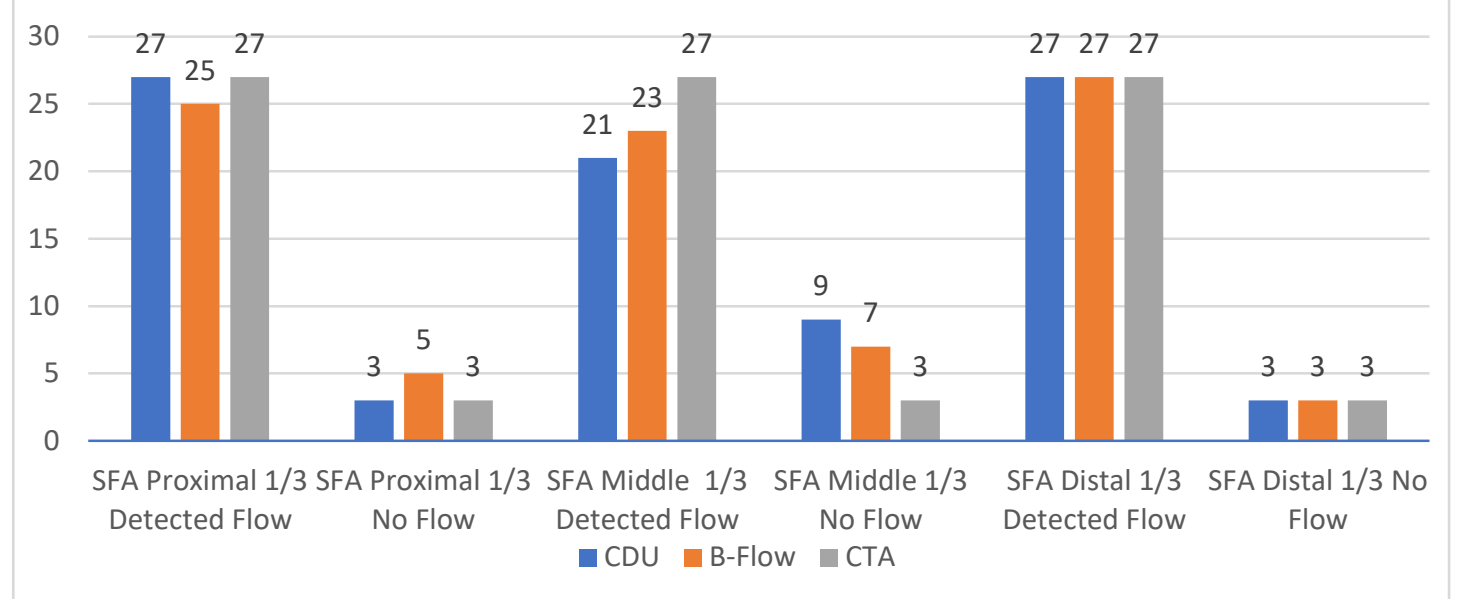

Graph 2. The results of the different segments of the SFA

- The incidence of detected flow at the SFA was $83.3 \%$ by both CDU and Bflow, while it was $90 \%$ by CTA.

- The incidence of no flow at the SFA was $16.6 \%$ by both CDU and B-flow, while it was $10 \%$ by CTA.

- The sensitivity of CDU at the SFA in our study was $100 \%$, the specificity was
$92.6 \%$, the positive predictive value was $60 \%$ and the negative predictive value was $100 \%$.

- The sensitivity of B-flow at the SFA in our study was $88.8 \%$, the specificity was $91.3 \%$, the positive predictive value was $53.3 \%$ and the negative predictive value was $98.6 \%$. 
Table 3. The results of the different segments of the Popliteal artery

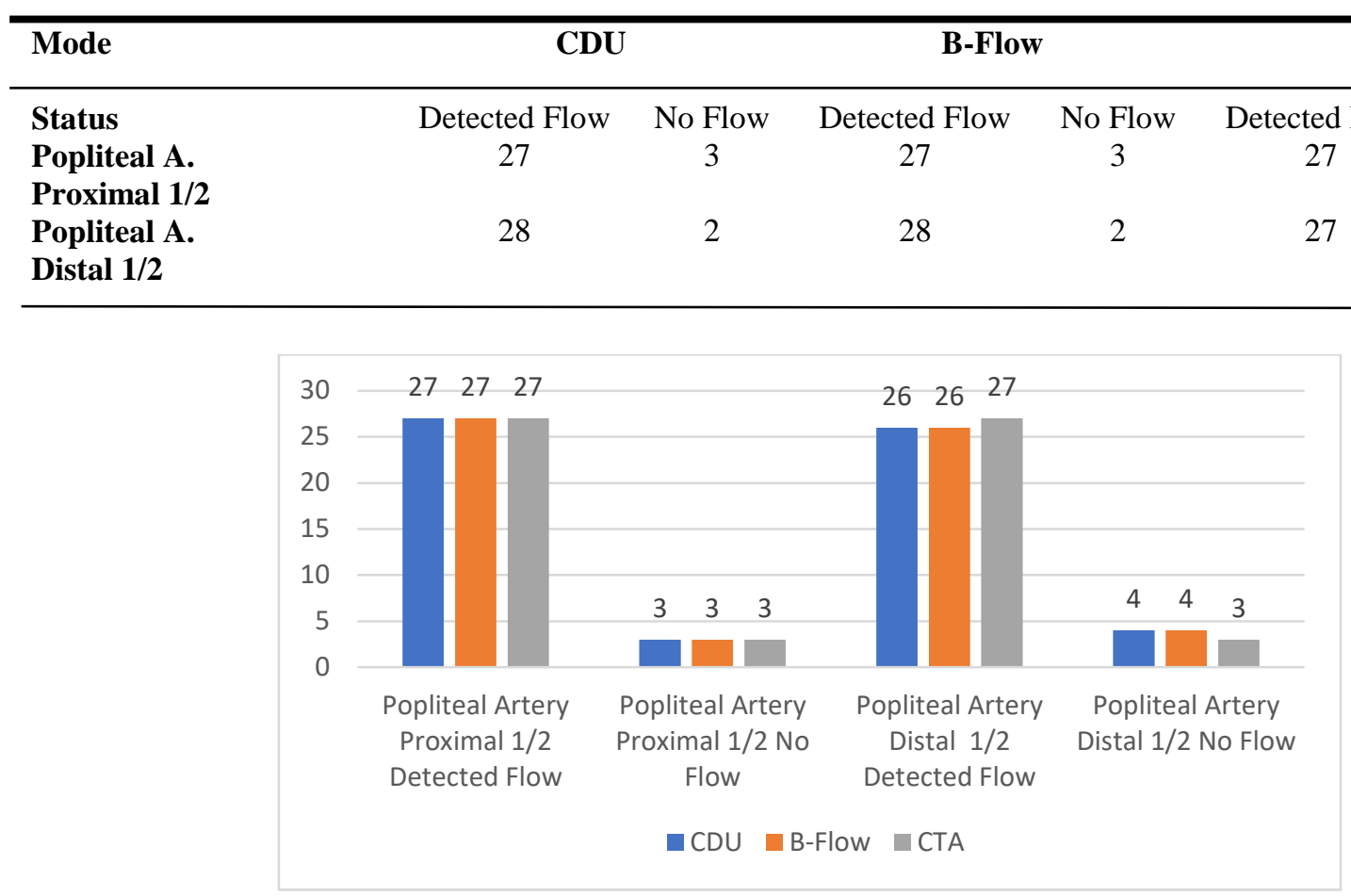

Graph 3. The Popliteal artery results

- The incidence of detected flow at the popliteal artery was $91.6 \%$ by both CDU and B-flow, while it was $90 \%$ by CTA.

- The incidence of no flow at the popliteal artery was $8.3 \%$ by both CDU and B-flow, while it was $10 \%$ by CTA.

- The sensitivity of CDU at the popliteal artery in our study was
$83.3 \%$, the specificity was $100 \%$, the positive predictive value was $100 \%$ and the negative predictive value was $98.2 \%$.

- The sensitivity of B-flow at the popliteal artery in our study was $83.3 \%$, the specificity was $100 \%$, the positive predictive value was $100 \%$ and the negative predictive value was $98.2 \%$. 
Table 4. The results of the different segments of the PTA

\begin{tabular}{lllllll}
\hline Mode & CDU & \multicolumn{2}{c}{ B-Flow } & \multicolumn{2}{c}{ CTA } \\
\hline Status & Detected Flow & No Flow & Detected Flow & No Flow & Detected Flow & No Flow \\
PTA Proximal 1/2 & 27 & 3 & 27 & 3 & 25 & 5 \\
PTA Distal 1/2 & 25 & 5 & 25 & 5 & 23 & 7 \\
\hline
\end{tabular}

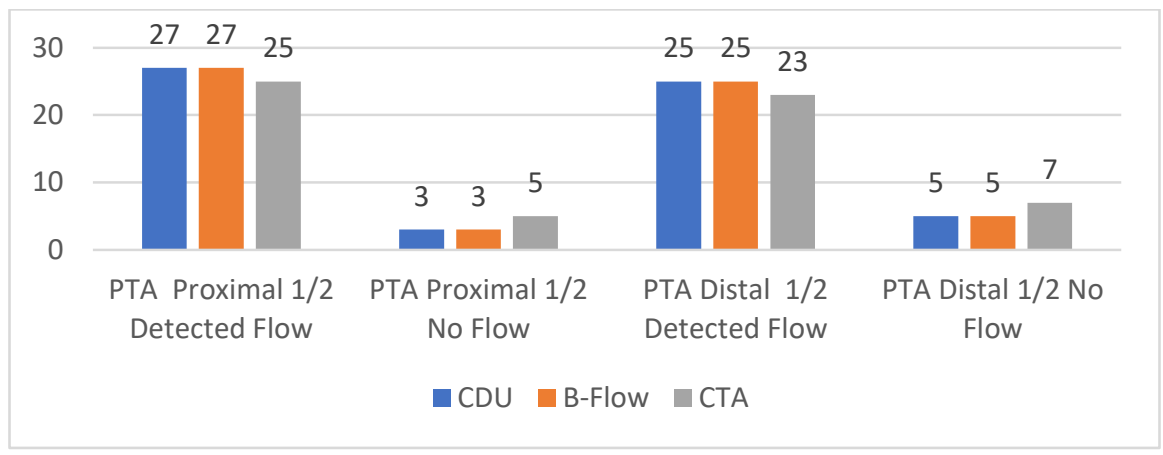

Graph 4. The results of the different segments of the PTA

- The incidence of detected flow at the PTA was $86.6 \%$ by both CDU \& Bflow, while it was $80 \%$ by CTA.

- The incidence of no flow at the PTA was $13.3 \%$ by both CDU \& B-flow, while it was $20 \%$ by CTA.

- The sensitivity of CDU at the PTA in our study was $58.3 \%$, the specificity was $97.9 \%$, the positive predictive value was $87.5 \%$ and the negative predictive value was $90.3 \%$.

- The sensitivity of B-flow at the PTA in our study was $58.3 \%$, the specificity was $97.9 \%$, the positive predictive value was $87.5 \%$ and the negative predictive value was $90.3 \%$.

Table 5. The results of the different segments of the ATA

\begin{tabular}{lcccccc}
\hline Mode & \multicolumn{2}{c}{ CDU } & \multicolumn{2}{c}{ B-Flow } & CTA \\
\hline Status & Detected Flow & No Flow & Detected Flow & No Flow & Detected Flow & No Flow \\
ATA Proximal 1/2 & 27 & 3 & 25 & 5 & 28 & 2 \\
ATA Distal 1/2 & 24 & 6 & 24 & 6 & 25 & 5 \\
\hline
\end{tabular}




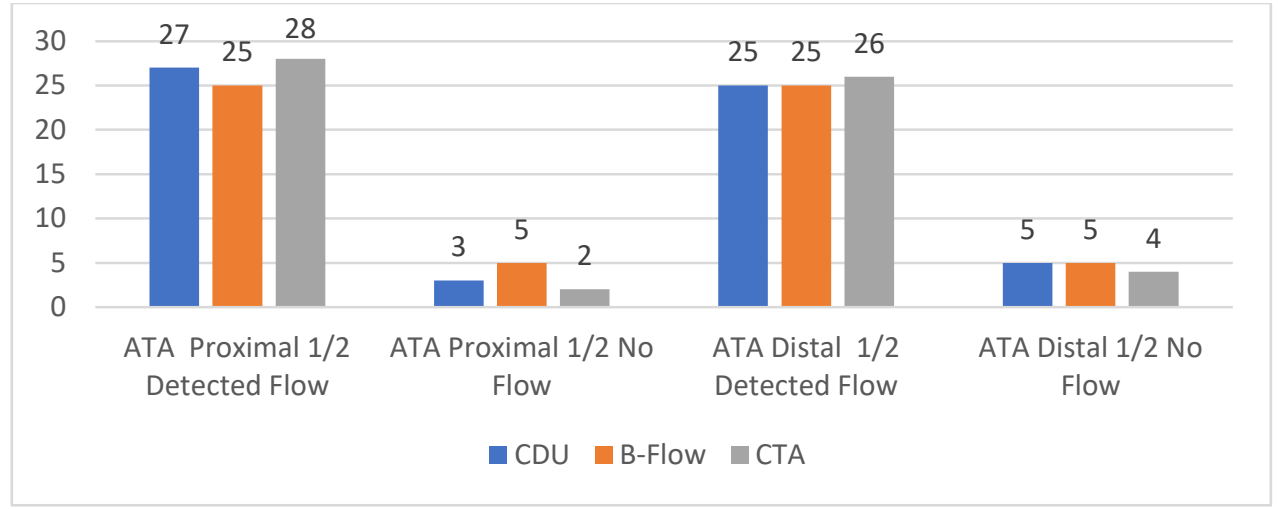

Graph 5. The results of the different segments of the ATA.

- The incidence of detected flow at the ATA was $85 \%$ by CDU, $81.6 \%$ by B-flow, while it was $88.3 \%$ by CTA.

- The incidence of no flow at the ATA was $15 \%$ by CDU, $18.3 \%$ by Bflow, while it was $11.6 \%$ by CTA.

- The sensitivity of CDU at the ATA in our study was $85.7 \%$, the specificity was $94.3 \%$, the positive predictive value was $66.6 \%$ and the negative predictive value was $98 \%$.

- The sensitivity of B-flow at the ATA in our study was $85.7 \%$, the specificity was $90.5 \%$, the positive predictive value was $54.5 \%$ and the negative predictive value was $97.9 \%$.

Table 6. The results of the different segments of the DPA.

\begin{tabular}{|c|c|c|c|c|c|c|}
\hline \multirow{2}{*}{$\frac{\text { Mode }}{\text { Status }}$} & \multicolumn{2}{|c|}{ CDU } & \multicolumn{2}{|c|}{ B-Flow } & \multicolumn{2}{|l|}{ CTA } \\
\hline & Detected Flow & No Flow & Detected Flow & No Flow & Detected Flow & No Flow \\
\hline DPA & 24 & 6 & 24 & 6 & 25 & 5 \\
\hline
\end{tabular}

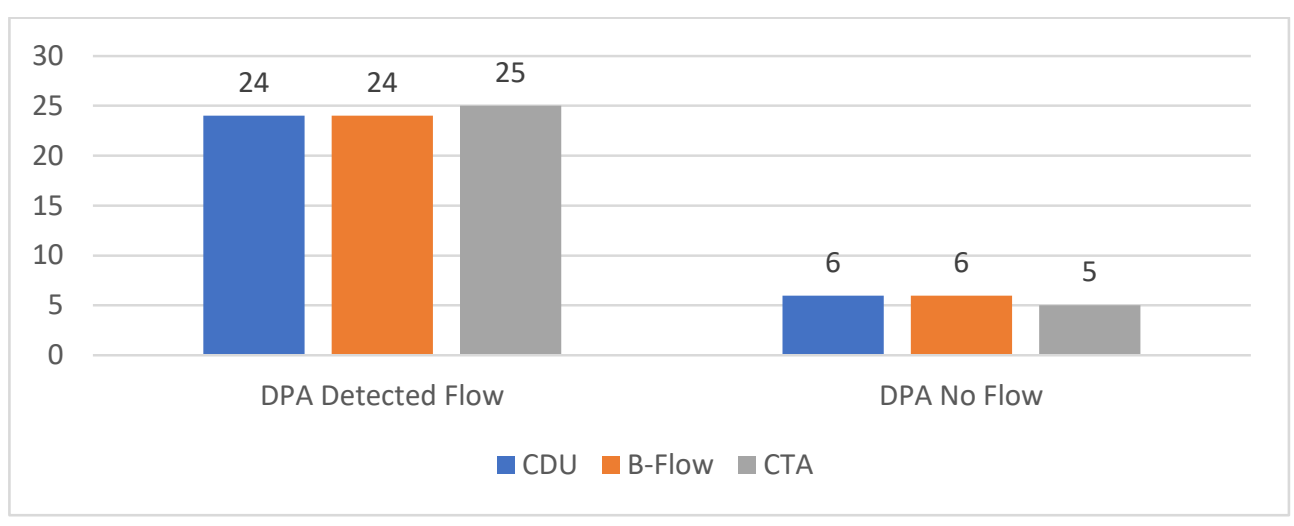

Graph 6. The results of the different segments of the DPA. 
- The incidence of detected flow at the DPA was $80 \%$ by CDU \& B-flow, while it was $83.3 \%$ by CTA.

- The incidence of no flow at the DPA was $20 \%$ by CDU \& B-flow, while it was $16.6 \%$ by CTA.

- The sensitivity of CDU at the DPA in our study was $100 \%$, the specificity was $96 \%$, the positive predictive value was $83.3 \%$ and the negative predictive value was $100 \%$.

- The sensitivity of B-flow at the DPA in our study was $100 \%$, the specificity was $96 \%$, the positive predictive value was $83.3 \%$ and the negative predictive value was $100 \%$.

\section{Discussion}

Firstly, BFI examination does not require separate equipment and it can be performed

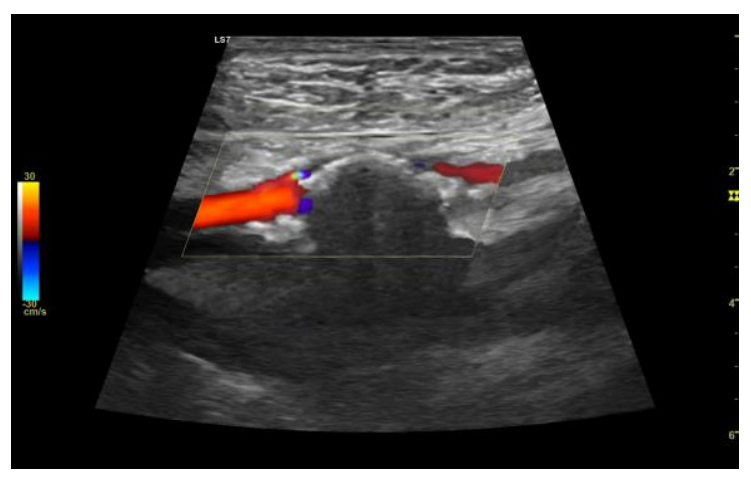

by using the same Doppler equipment which is advantageous in terms of time and cost.

As for our comparative study, BFI at the CFA showed better results than CDU. The densely calcified plaques cause posterior acoustic shadowing artifacts that obscure luminal details. However, this shadowing artefact was often less pronounced during $\mathrm{BFI}$, allowing the visualization of a residual patent lumen that could not be seen using CDU alone; and as such, spectral Doppler could now be sampled in regions that were otherwise obscured (Figure 1). Moreover, visualizing the plaque surface was more satisfactory during BFI, because it has higher spatial resolution, and it is exempt from movement, blooming and aliasing artifacts; with a better chance to show excavations that might been associated with an increased risk of developing thromboembolic events (Figure 2) ${ }^{6}$.

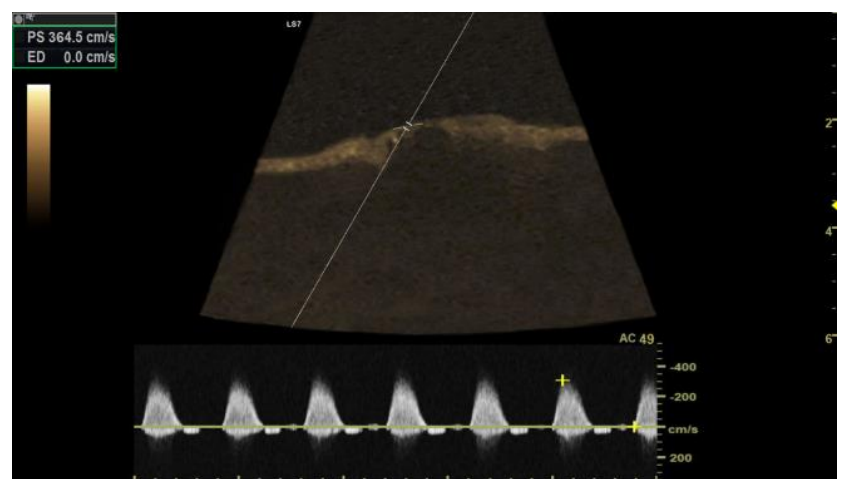

Figure 1: a) CDU showing a large dense plaque at the CFA obscuring blood flow with no residual lumen can be seen. b) BFI at the CFA showing the residual patent lumen. spectral Doppler can now be applied to regions that showed no flow by CDU. 
At the different segments of SFA, BFI failed to detect any flow with markedly obese patients, as the boosted sent signals are scattered by the subcutaneous fat, beside that the arteries are deeply located. As for CDU, which has better penetration, detecting flow could be done (Figure 3). On the other hand, BFI showed higher discrimination than CDU in presence of densely calcified plaques with regular patients, despite not being completely free of its acoustic shadowing effects. In some cases, no flow could be visualized within the SFA using either mode, although CTA proved their patency.

At the different segments of popliteal artery. Both CDU and BFI displayed similar results. A collateral artery feeding the TPT was mistaken for the main popliteal artery.

At the PTA segments, both modes displayed similar results. A congenital absence and a thrombosed PTA, replaced by collateral branches, were mistaken for a patent PTA. Both modes as well failed to detect flow in some cases at the distal segments with densely calcified plaques, although CTA proved their patency.

At the proximal half of ATA, CDU showed better results than BFI in detecting flow in presence of remarkable subcutaneous edema, as BFI has poor penetration; however, it was easier in regular patients to detect sites of collaterals take off by BFI, thanks to its angle independency and the extended FOV (Figures $4 \& 5$ ). At the distal half of ATA, both modes displayed similar results, a densely calcified artery with low flow was mistaken to be thrombosed, proved to be patent by CTA.

At the DPA, both modes displayed similar results. a densely calcified artery with low flow was mistaken to be thrombosed, proved to be patent by CTA as well.
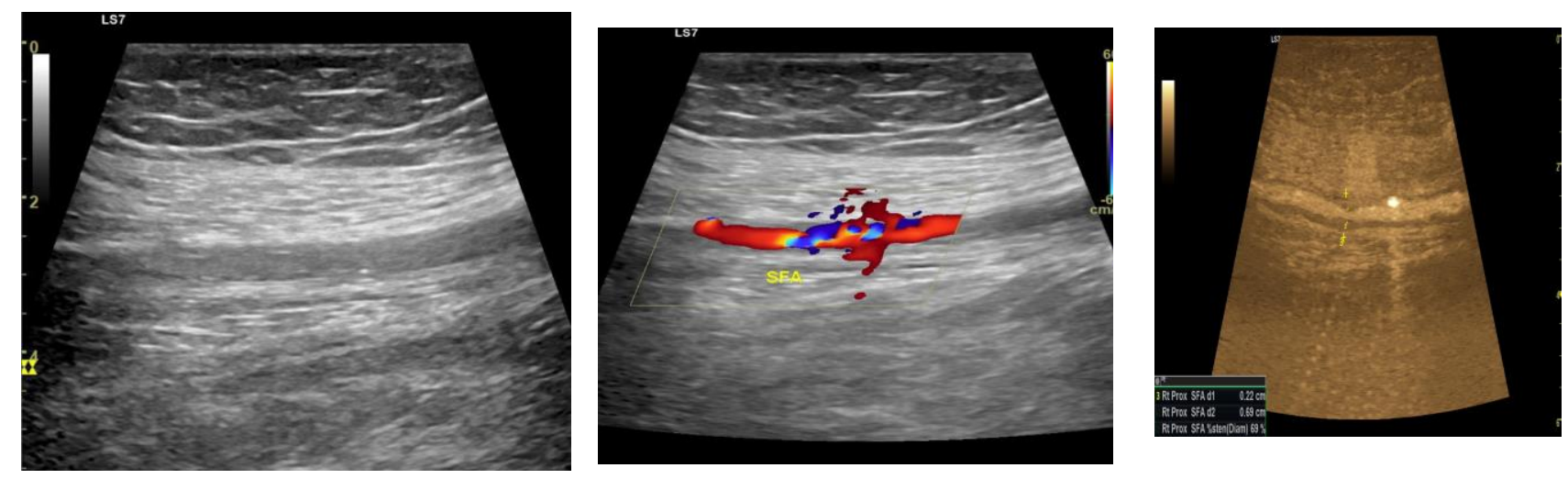

Figure 2: a) B-mode of the SFA with no clear demarcation between the soft plaque and the preserved lumen. b) CDU showing aliasing denoting the presence of a stenosis. c) B-flow examination with preservation of background echoes the stenotic segment can be clearly identified from the blood flow column. 

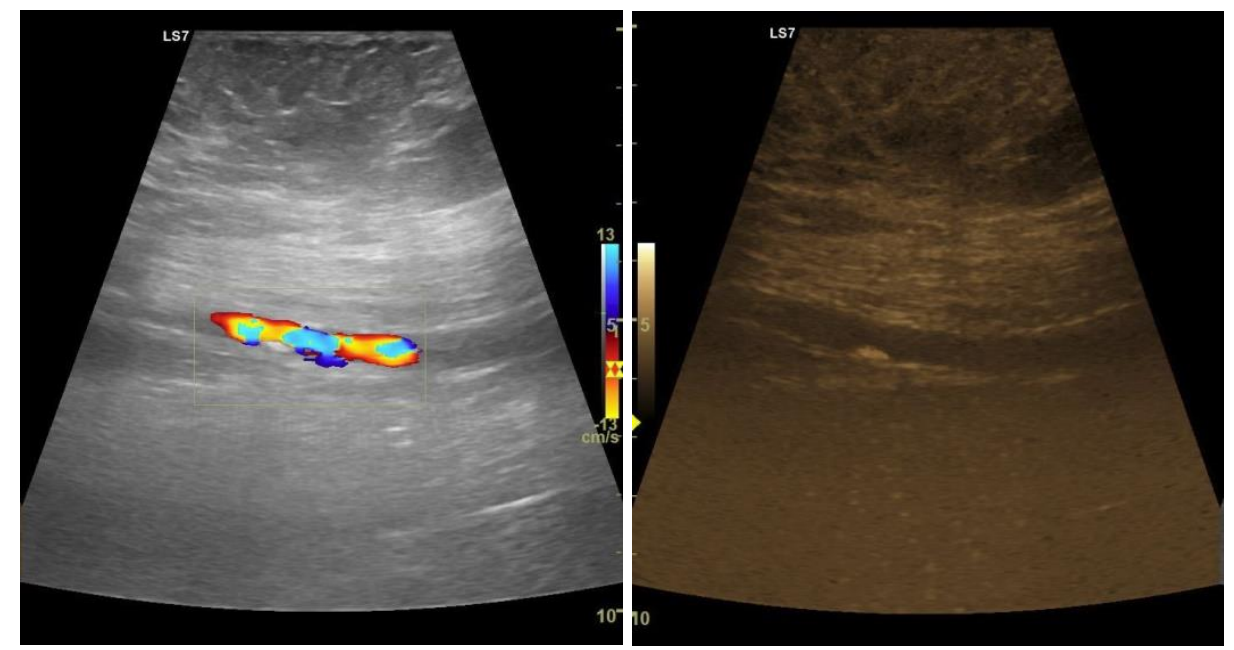

Figure 3: a) CDU in a markedly obese patient showing a small dense plaque at the proximal segment of the right SFA with no aliasing can be seen. b) B-Flow mode with preservation of background echoes showing a small plaque. No flow can be seen however.

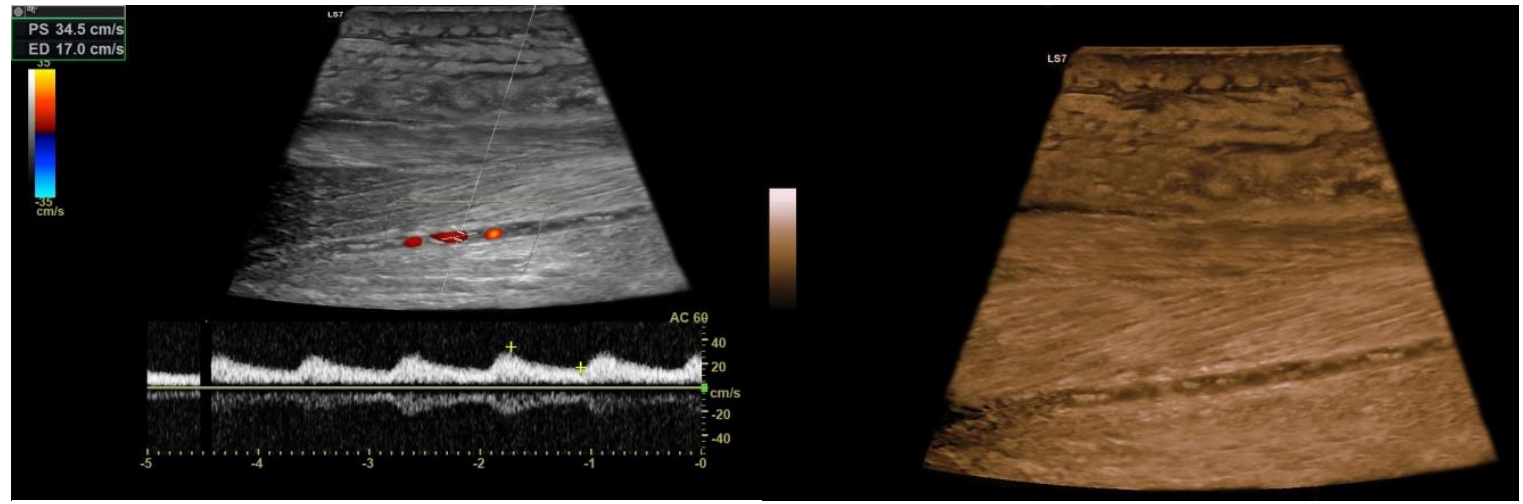

Figure 4: a) CDU with spectral Doppler in a patient with prominent subcutaneous edema and densely calcified ATA showing beaded color flow and tardus parvus waveform. b) B-flow mode with preservation of background echoes; however, no blood flow can be seen

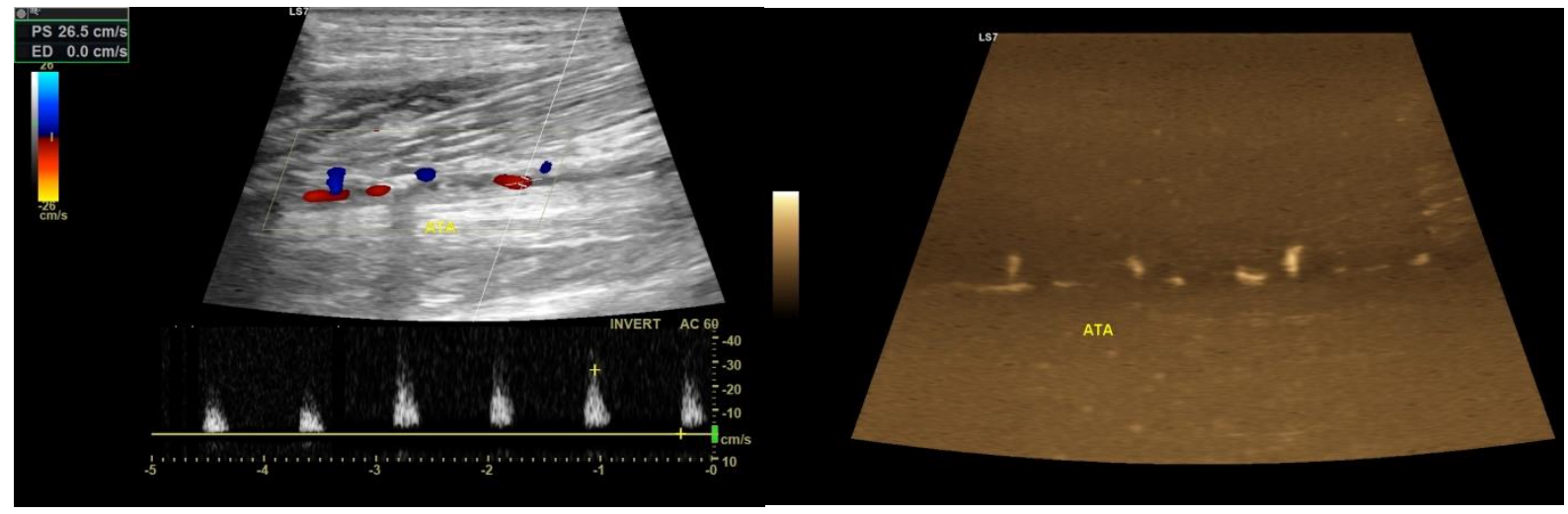

Figure 5: a) CDU of the ATA showing beaded color flow and a single collateral artery can be visualized within the color box. b) BFI displays multiple defects within the blood flow column and multiple collaterals can be seen at the same time 
Generally speaking, BFI introduced the plaque surface. However, it is not free additional features promoting the noninvasive methods of diagnosing PAD. It has from limitations like extensive plaque higher discrimination in presence of densely calcified plaques, better in detecting sites of collaterals take-off, and better in visualizing

Table 7. Brief comparison of different imaging modes (Rating 1-3, 1 is least and 3 is most desirable) ${ }^{6}$

\begin{tabular}{lcc}
\hline Color Display of Flow Direction & Yes & No \\
Display Flow Velocity & Yes & No \\
Aliasing & Yes & No \\
Region of Interest & Yes & No (extended FOV) \\
Spatial Resolution & 1 & 3 \\
Hemodynamics & 1 & 3 \\
Presence of Color Overwrite & 1 & 3 \\
Flow Angle Dependence & 1 & 3 \\
Background Image Quality & 3 & 2 \\
Penetration & 3 & 1 \\
\hline
\end{tabular}

Concordant results of CDU at the FemoroPopliteal segments show better sensitivity, slightly better specificity, much lower PPV and higher NPV. Concerning the infraPopliteal segments, the concordant results show similar sensitivity, slightly higher specificity \& PPV, and a much higher NPV. Unfortunately, BFI lacks similar studies and no comparative data could be found.

Finally, this study included a low number of cases, and the lacks of other comparative studies represent other further limitations. Therefore, more prospective comparative studies with gold standard imaging modalities are required to clarify the sensitivity and specificity of B-Flow in study of PAD.

\section{Conclusion}

At the femoral segments, BFI has higher diagnostic accuracy than CDU in presence of densely calcified plaques. As for the infrapopliteal segments, CDU displayed better results; however, BFI was better in 
detecting sites of collaterals take off. Unfortunately, BFI is not free of limitations like depth and subcutaneous edema. As such, we do not encourage the use of BFlow as a sole ultrasound imaging mode, but as a complementary technique to use in situations where CDU findings are inconclusive or unclear, providing better diagnostic accuracy than either method alone.

\section{References}

1. Michal Tendera, Victor Aboyans, Marie-Louise Bartelink, Iris Baumgartner, Denis Clément, Jean-Philippe Collet, et al. ESC Guidelines on the diagnosis and treatment of peripheral artery diseases. Additional value of b-flow imaging in arterial wall calcifications covering atherosclerotic disease of extracranial carotid and vertebral, mesenteric, renal, upper and lower extremity. Medical University of Silesia, Ziolowa (EDS). March 2017.

To cite this article: Mahmoud H. ElShenawy, Islam M. ElShazly, Hesham E. ElSheik. The Added Value of B-Flow Technique in Assessment of Lower Limb Arterial Lesions with Densely Calcified Atheromatous Plaques. BMFJ 2021; 38(1): 280-293. DOI: 10.21608/bmfj.2021.38713.1304
2. Chiao R Y, Mo L Y, Hall A L. B-mode blood flow (B-flow) imaging. GE Med Syst Ultrasonics Symposium,IIE. 2000

3. Mazzariol F. Values and limitations of duplex ultrasonography as the sole imaging method of preoperative evaluation for popliteal and infrapopliteal bypasses. Ann Vasc Surg. 1999;13:1-10,.

4. Henri P, Tranquart F. B-flow ultrasonographic imaging of circulating blood. $\mathrm{J}$ Radiol. 2000;81:465.

5. Weskott HP. B-flow: a new method for detecting blood flow. Ultraschall Med 2000; 21:59-65

6. Viles-Gonzalez J F, Fuster V, Badimon J J. Atherothrombosis. a widespread disease with unpredictable and life-threatening consequences. Eur Heart J. 2004;25:1197. 